\title{
HSV and Dual Tree Complex Wavelet Packet Transform based Image fusion for Satellite Images
}

\author{
Dr.G. Dheepa ${ }^{* 1}$, Dr. S. Sukumaran ${ }^{2}$ \\ ${ }^{I}$ (Assistant Professor, Department of Computer Science, PKR Arts College for Women,Gobi, Tamilnadu, India) \\ ${ }_{2}^{2}$ (Associate Professor, Department of Computer Science, Erode Arts and Science College, Tamilnadu, India.)
}

\begin{abstract}
Image fusion is the process of integrating two or more images of a specific scene, captured from different sensors to form a single image that contains all the details of the source images. In satellite remote sensor images, this technique is used in the integration of the geometric detail of a high-resolution panchromatic (PAN) image and the spectral information of a low resolution multi -spectral (MS) image to form a single high resolution multispectral image. For satellite images, wavelet based fusion methods have shown to produce better results. The core objective of this paper is to introduce a new approach by using DualTreeComplex Wavelet Packet Transform (DT-CWPT) and HSV to fuse PAN image and MS image. The merits of using DT-CWPT over Discrete Wavelet Packet Transform (DWPT) and Dualtree Complex Wavelet Transform (DT-CWT) are briefed. Then DT-CWPT and its properties are explained and a new fusion method using DTCWPT and HSV is proposed. Finally evaluation of experimental results using various quality assessment metrics shows that the proposed method performs remarkably better than the other existing wavelet based fusion methods.
\end{abstract}

Keywords: Remote sensing, Image fusion, Discrete wavelet packets, Dual Tree Complex Wavelet Packet Transform.

\section{Introduction}

In optical remote sensing, several commercial earth observation satellites such as SPOT, Ikonos, Quickbird, etc...carry dual-resolution sensors, which collect panchromatic image and multispectral images at different spatial resolutions. A PAN image gives detailed geometric features, while the MS images contain richer spectral information. An effective way to utilize both the images is to fuse the PAN and MS image to form a single high resolution multispectral image. This would be useful in many applications such as environmental/ agriculture assessment, urban mapping, monitoring and sustainable management of natural resources change detection [2].

Some preprocessing steps are to be performed before fusing the images. A geometric registration and a radiometric adjustment of the images to one another are to be done. Since, the PAN image has a different spatial resolution from that of MS images, resampling is done to bring the MS images to the same size of PAN. In satellite image fusion, wavelet transform based fusion methods are widely used and found to give better results. The advantage of wavelet transform is that it can analyze signal in both frequency domain and time domain.

For a given signal, the frequency decomposition provided by the classical discrete wavelet transform (DWT) might not be optimal. The discrete wavelet packet transform (DWPT) provides a dictionary of bases over which one can search for an optimal representation for the signal. But, both the DWT and the DWPT suffers from two major drawbacks: lack of shift invariance and poor directional selectivity for diagonal features. The dualtree complex wavelet transform (DT-CWT), introduced by Kingsbury [1] overcomes these two limitations.

\section{Dual tree Complex Wavelet Transform}

The DT-CWT employs two real DWTs; the first DWT gives the real part of the transform while the second DWT gives the imaginary part as shown in Fig 1. They use two different sets of filters which are jointly designed so that the overall transform is approximately analytic.

\footnotetext{
* Corresponding author
} 


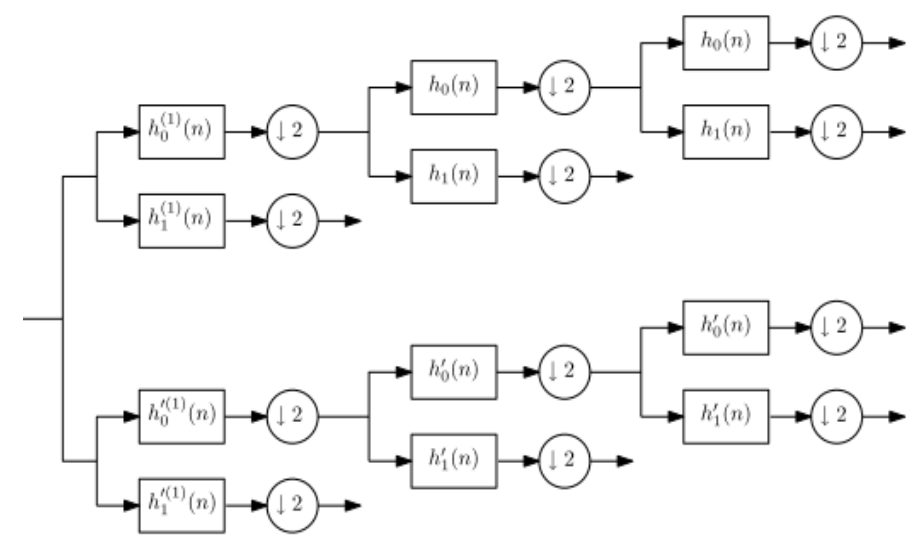

Fig 1. Three level complex dual tree using two FBs operating in parallel.

The wavelet associated with the first wavelet filter bank (FB) is denoted as $\psi(t)$ and the wavelet associated with the second FB as $\psi^{\prime}(t)$. The wavelet $\psi(t)$ is defined by

$$
\psi(t)=\sqrt{2} \sum_{n} h_{1}(n) \phi(2 t-n)
$$

where

$$
\phi(t)=\sqrt{2} \sum_{n} h_{0}(n) \phi(2 t-n)
$$

The second wavelet, $\psi^{\prime}(t)$, is defined similarly in terms of $\left\{h_{0}^{\prime}(n), h_{1}^{\prime}(n)\right\}$. For the ideal DT-CWT, the second wavelet, $\psi^{\prime}(t)$, is the Hilbert transform of the first wavelet, $\psi(t)$,

$$
\psi^{\prime}{ }_{1}(t)=H\{\psi(t)\}
$$

If the low-pass filter $h^{\prime}{ }_{0}(n)$ is equal to the half-sample delayed version of $h_{0}(n)$ then the wavelets generated by the DT-CWT satisfy the above equation.

In DT-CWT, shift invariance can be obtained by using two parallel wavelet trees but, they are subsampled differently, which leads to the lack of frequency localization. The disadvantages of DT-CWT are overcome in DualTree Complex Wavelet Packet Transform (DT-CWPT).

\section{Dual tree Complex Wavelet Packet Transform}

To construct the packet form of the DT-CWT (DT-CWPT), each of the subbands should be repeatedly decomposed using low-pass/high-pass perfect reconstruction (PR) FBs. The PR FBs should be chosen so that the response of each branch of the second wavelet packet FB is the discrete Hilbert transform of the corresponding branch of the first wavelet packet FB. Then each subband of the DT-CWPT will be analytic.

The PR FB which is used to decompose the first FB of the DT-CWT should also be used decompose the second (dual) FB - in order to preserve the Hilbert transform relationship already satisfied by those branches. The branches of the DT-CWT that do not already satisfy the Hilbert transform property are the lowpass branch of the final stage and the high-pass branch of the first stage. The low-pass branch of the final stage is not further decomposed.

The high-pass branch of the first stage, $\quad h_{1}^{(1)}(n)$ and $h_{1}^{\prime(1)}(n)$ satisfy $h_{1}^{\prime(1)}(n)=h_{1}^{(1)}(n-1) \quad$ which is exactly the same relationship satisfied by the low-pass filters of the first stage, $h_{0}^{\prime(1)}(n)=h_{0}^{(1)}(n-1)$. The FB structure following the low-pass filters of the first stage should also follow the high-pass filters of the first stage. This procedure produces a DT-CWPT consisting of two wavelet packet FBs operating in parallel, where some filters in the second wavelet packet FB are the same as those in the first wavelet packet FB. The first of these two wavelet packet FBs is illustrated in Fig. 2, for a three-stage DT-CWPT.

In Figure 2, $\mathrm{H}_{0}{ }^{(1)}$ and $\mathrm{H}_{1}{ }^{(1)}$ represent the approximation and detail coefficients of the upper and lower tree respectively. They are further split into corresponding approximation coefficients $\mathrm{H}_{0}$ and detail coefficients $\mathrm{H}_{1}$. The approximation coefficients $\mathrm{H}_{0}$ are further split into approximation $\left(\mathrm{H}_{0}\right)$ and detail $\left(\mathrm{H}_{1}\right)$. Similarly, detail coefficients $\mathrm{H}_{1}$ are further split into approximation $\left(\mathrm{F}_{0}\right)$ and detail $\left(\mathrm{F}_{1}\right)$. This process is repeated for the required levels of decomposition. The second wavelet packet FB is obtained by replacing the first stage filters $h_{i}^{(1)}(n)$ by $h_{i}^{(1)}(n-1)$ and by replacing $h_{i}(n)$ by $h_{i}^{\prime}(n)$ for $i \in\{0,1\}$. The filters $f_{i}(n)$ are those that must be used in both FBs. 


\subsection{Properties of DT-CWPT}

- Approximate Shift-invariance

In DT-CWPT, the energy in each subband is approximately preserved if the input sequence is shifted by an arbitrary number of samples. Because it is nearly shift-invariant, best-frame algorithms using the DTCWPT are more robust to signal translation than best-basis algorithms using the real DWPT. The approximate shift-invariance property is due to the reduction in aliasing which is because the band-pass response of each branch is approximately analytic.

- Best Basis Selection

Allowing the iteration of the high-pass outputs as well as the low-pass outputs, the DWPT introduces a number of different trees to analyze the input. Each tree yields a unique frequency decomposition of the input. To express the input in the most compact way, the search for a best basis (the one that provides a representation of the input signal minimizing a cost function) is done among the collection of trees. A basis selection algorithm is used to choose among the bases that the DT-CWPT provides, by adapting the basis selection algorithm in [5]. DT-CWPT is a fixed linear transform and necessitates only the design of DT-CWT filters and so there is no complexity in the search algorithm. The DT-CWPT may be extended to 2-D similar to the DT-CWT [20].

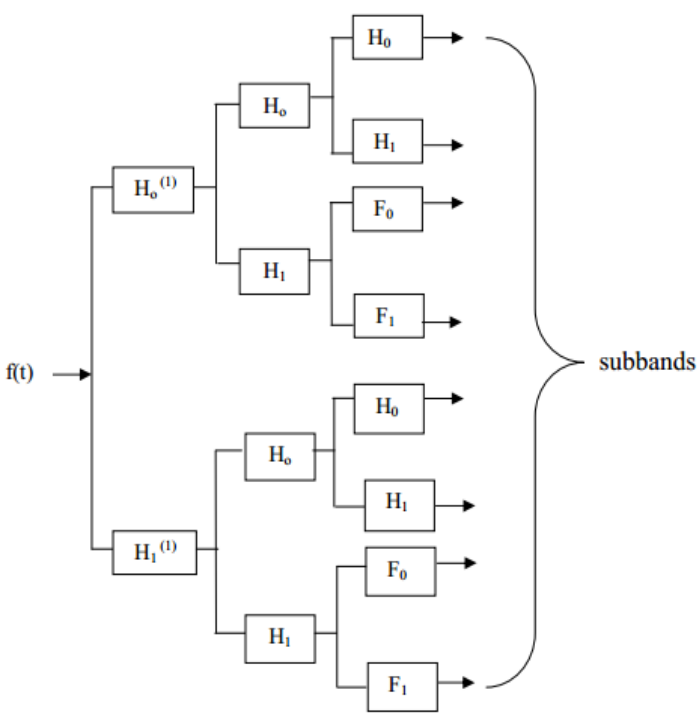

Fig 2. The first wavelet packet FB of a three-stage DT-CWPT.

Since the limitations in DWPT and DT-CWT are overcome by DTCWPT, it is considered as a better alternative to use it for fusing satellite images.

\section{Proposed Fusion Method Using HSV Transform And DT-CWPT}

The hexcone transform of IHS is referred to as HSV method which derives its name from the parameters, Hue, Saturation, and Value. HSV method transforms the R, G and B bands of the MS image into HSV components. The Value component is replaced by the PAN image and inverse transform is performed to obtain a high spatial resolution MS image. This is one of the most widely applied fusion procedure for merging panchromatic imagery with three-color multispectral imagery. The problem with the HSV method is that spectral distortion occurs during the fusion process. For QuickBird and Ikonos images the color distortion is especially significant.

The wavelet based fusion method can well retain the color information but the spatial detail from a PAN is often different from that of a MS band having the same spatial resolution because of their spectral range difference which introduces some color distortion into the fusion results. Also the integration between color and spatial details appear unnatural. So in order to overcome the limitations and utilize the merits of both methods, we integrate HSV and DT-CWPT technique to form a new fusion method as shown in Fig 3.

As a preprocessing step, both the PAN and MS images are registered geometrically so that both have the same size. The fusion procedure is described as follows:

1. Using HSV transform the MS image is transformed to HSV components.

2. The PAN image is histogram matched to that of the $\mathrm{V}$ component to get a new Pan image.

3. The new Pan image and V component are decomposed into several sub bands using DT-CWPT to obtain 
complex wavelet packet coefficients.

4. The LL subbands block shows the image's approximate characteristic. Using substitutive scheme, the low frequency parts of $\mathrm{V}$ component are replaced by the corresponding parts of PAN image.

5. The high frequency parts are fused using the maximum selection rule to give an intermediate fusion image. In this way the fusion takes place in all the decomposition levels and the more dominant features at each scale are preserved in the new combined wavelet packet representation.

6. Inverse DT-CWPT is applied to obtain an intermediate fusion image which has the spatial detail of PAN image and has a high correlation (similar grey value distribution) with the Value component of HSV transform.

7. The $\mathrm{V}$ component is replaced with the intermediate fusion image. Inverse HSV transform is applied to transform the HSV components back to RGB space to get the final fused image.

\section{Experimental Results}

The Quickbird satellite images of 0.61-m panchromatic and the Red, Green, Blue and Near Infrared bands of the 2.4-m resolution multispectral images are used as experiment data. To completely utilize the available wave bands information, and match the wavelength range of the participating bands in fusion band 4 , band 3 and band 1 was for R, G, B, respectively. The $2.4 \mathrm{~m}$ resolution multi-spectral bands are resampled to $0.61-\mathrm{m}$ pixel size before fusion is done. The original panchromatic image and resampled MS image are shown in Fig.4 and Fig.5 respectively. The resampled multispectral and Pan bands are then fused using DWT, DWPT, DT-CWT and the proposed method. The corresponding fusion results are shown in Fig.6a, Fig 6b, Fig.6c and Fig.6d.

\section{Result Analysis}

By comparing the results visually, the DWT method blends the spatial information of PAN and spectral information of MS image. Color distortion appears significantly when compared to the original MS image. The DWPT and DT-CWT method enhances the spatial information and color information but still a little color distortion exits which is apparently visible. The proposed fusion method integrates the spatial information of the PAN and spectral information of the MS image into a single fused image very well. The colors in the fusion result look close to that of the original MS images, and spatial details as detailed as the original PAN image. It well preserves both the spatial and original spectral content. To evaluate the performance of each fusion method quantitatively a statistical comparison is done. Mathematical methods were used to judge the quality of fused image in respect to their improvement of spatial resolution while preserving the spectral content of the data. The correlation coefficient is most widely used similarity metric. Another commonly used assessment metric is the Root Mean Square Error (RMSE). Multimodal statistical indices such as UIQI and ERGAS have also been calculated to compare the fusion results. 


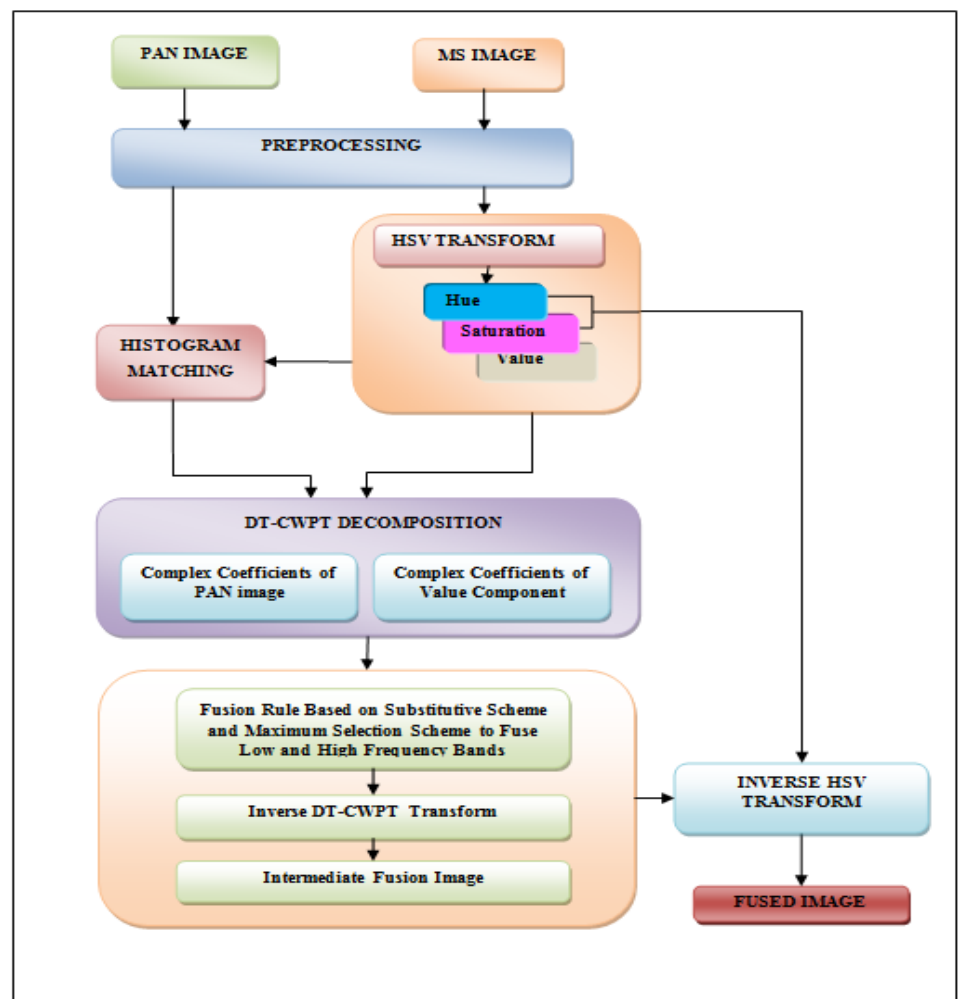

Fig 3. Flow scheme of proposed fusion method using HSV transform and DT-CWPT

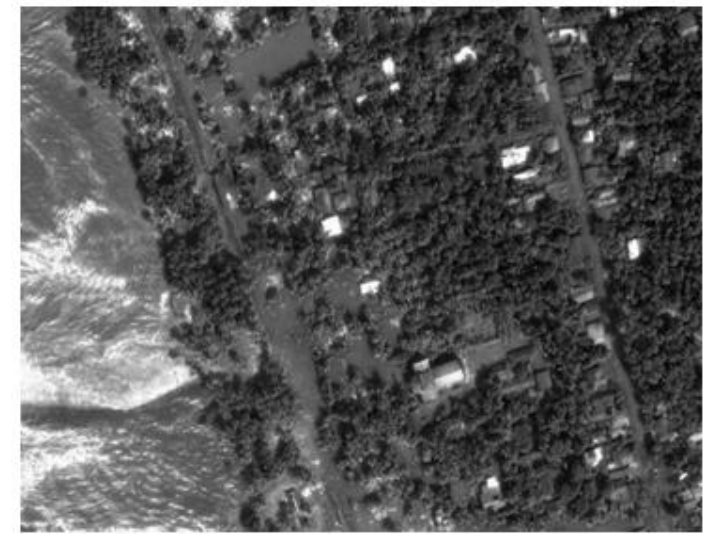

Fig. 4. Original panchromatic image

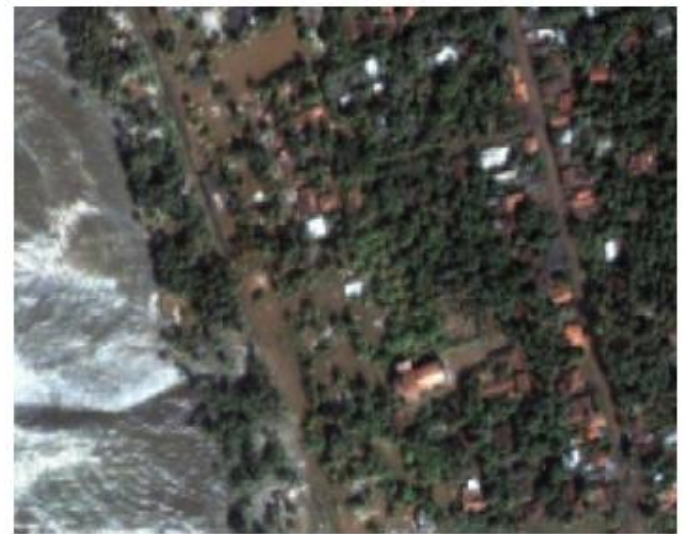

Fig. 5. Resampled multispectral image

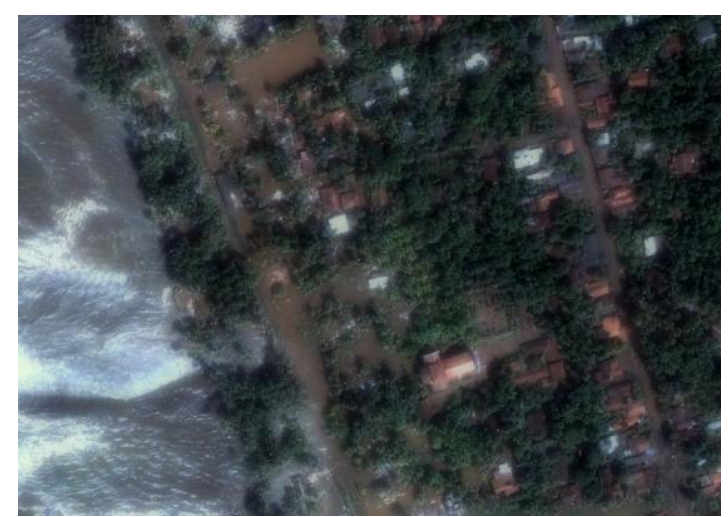

Fig. 6a. DWT fusion result 


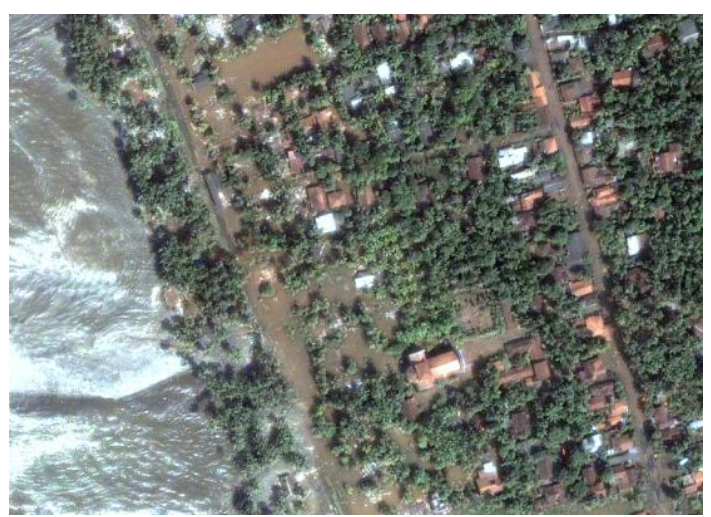

Fig. 6b. DWPT fusion result

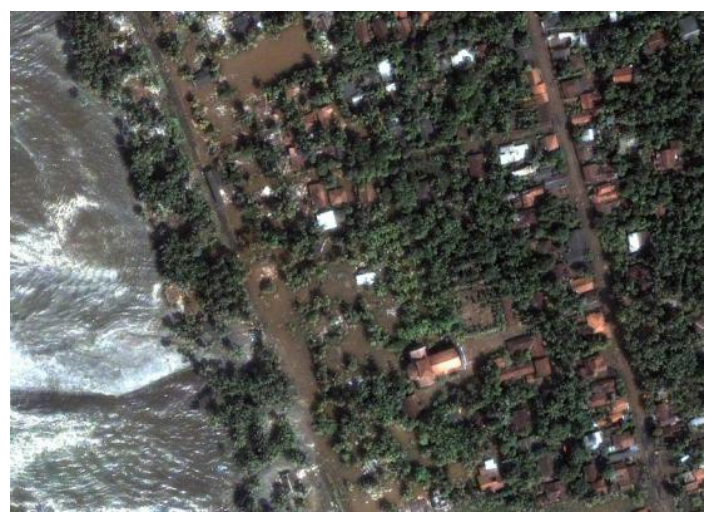

Fig. 6d. Proposed DT-CWPT Fusion Result

$>$ Correlation Coefficient (CC)

The correlation coefficient measures the closeness or similarity in small size structures between the original and the fused images. It ranges from -1 to +1 .Values close to +1 indicates that they are highly similar while the values close to -1 indicate that they are highly dissimilar.

$$
C C=\frac{\sum_{i=1}^{N} \sum_{j=1}^{N}\left(M S_{i, j}-\overline{M S}\right)\left(F_{i, j}-\bar{F}\right)}{\sqrt{\sum_{i=1}^{N} \sum_{j=1}^{N}\left(M S_{i, j}-\overline{M S}\right)^{2} \sum_{i=1}^{N} \sum_{j=1}^{N}\left(F_{i, j}-F\right)^{2}}}
$$

where $C C$ is the Correlation Coefficient, $F$ is the fused image and $i$ and $j$ are pixels, $M S$ is the multispectral data.

$>$ Root mean square error (RMSE)

The RMSE was computed from the standard deviation and the mean of the fused and the original image.

$$
R M S E=\sqrt{\left(\sigma_{M S}-\sigma_{F}\right)^{2}+\left(M_{M S}-M_{F}\right)^{2}}
$$

where $\sigma_{M S}$ is standard deviation of multispectral image, $\sigma_{F}$ is standard deviation of fused image; $M_{M S}$ equals to mean value of multispectral image, and $M_{F}$ equals to mean value of multispectral image. The best possible value is zero.

\section{ERGAS}

ERGAS is the abbreviation of Erreur Relative Globale Adimensionnelle de Synthèse (Relative global dimensional error).It calculates the amount of spectral distortion and the formula is given by:

$$
E R G A S=100 \frac{h}{l} \sqrt{\frac{1}{N} \sum_{i=1}^{N}\left[\frac{R M S E\left(B_{i}\right)^{2}}{\left(M_{i}\right)^{2}}\right]}
$$

where $N$ is the number of bands involved in fusion, $h / l$ is the ratio of the spatial resolution of original Pan and MS images. $M_{i}$ is the mean value for the original spectral image $B_{i}$. ERGAS values larger than 3 stands for 
synthesized images of low quality, while less than 3 represent a satisfactory quality [20].

$>$ Universal Image Quality Index (UIQI)

The UIQI [13] measures how much of the salient information contained in reference image is transferred to the fused image. UIQI is devised by considering loss of correlation, luminance distortion and contrast distortion. The range of this metrics varies from -1 to +1 and the best value is 1 .

$$
U I Q I=\frac{\sigma_{A B}}{\sigma_{A} \sigma_{B}} \cdot \frac{2 \mu_{A} \mu_{B}}{\mu_{A}^{2}+\mu_{B}^{2}} \cdot \frac{2 \sigma_{A} \sigma_{B}}{\sigma_{A}^{2}+\sigma_{B}^{2}}
$$

where $\sigma$ represents the standard deviation and $\mu$ represents the mean value. The first term in RHS is the correlation coefficient, the second term represents the mean luminance and the third measures the contrast distortion. The range of this metrics varies from -1 to +1 and the best value is 1 .

The quantitative evaluation results are shown in Table.1. To simplify the comparison of the different fusion methods, the values of quantitative indicators CC, RMSE, ERGAS and UIQI of the fused images are provided as chart in Fig.7a, Fig.7b, Fig.7c and Fig.7d respectively.

Table 1. Evaluation Results of Fusion Methods

\begin{tabular}{|l|c|c|c|c|}
\hline \multicolumn{1}{|c|}{$\begin{array}{c}\text { Methods / } \\
\text { Assessment Indices }\end{array}$} & DWT & DWPT & DT-CWT & DT- CWPT \\
\hline CC & 0.8943 & 0.9312 & 0.9432 & 0.9621 \\
\hline RMSE & 0.8675 & 0.5013 & 0.4932 & 0.4718 \\
\hline ERGAS & 2.8943 & 2.4128 & 2.3217 & 2.1325 \\
\hline UIQI & 0.7998 & 0.8523 & 0.9043 & 0.9175 \\
\hline
\end{tabular}

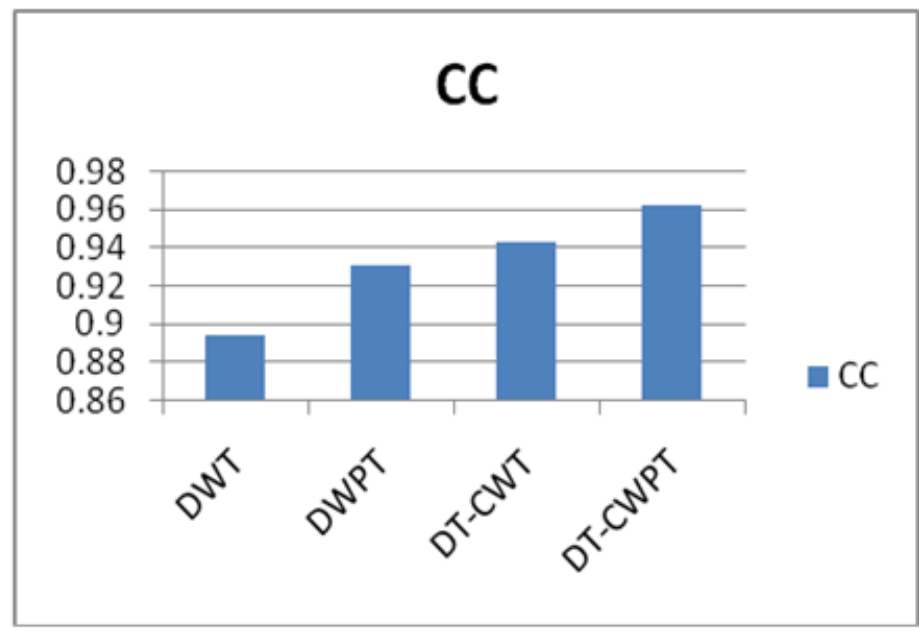

Fig .7a Correlation Coefficient for Fusion Methods

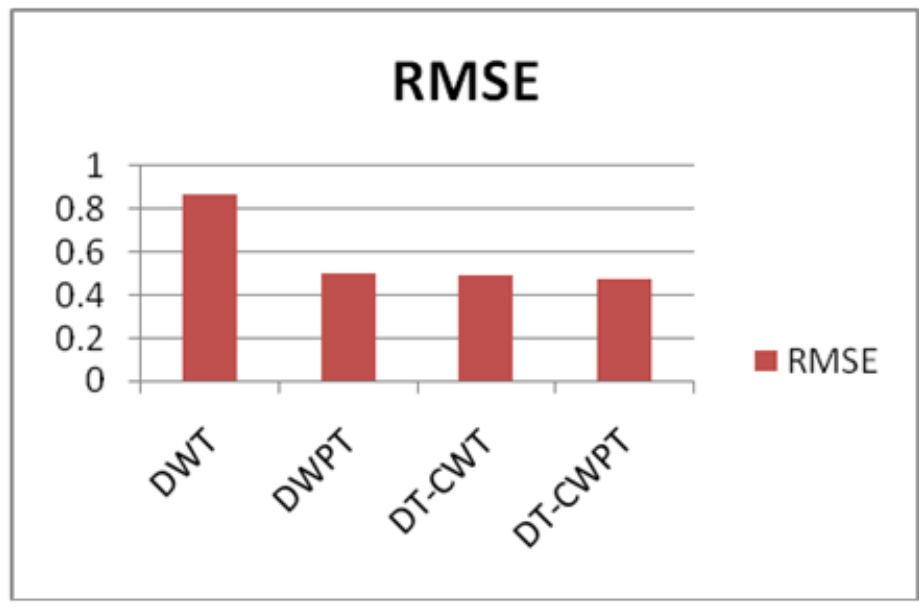

Fig. 7b RMSE Value for Fusion Methods 


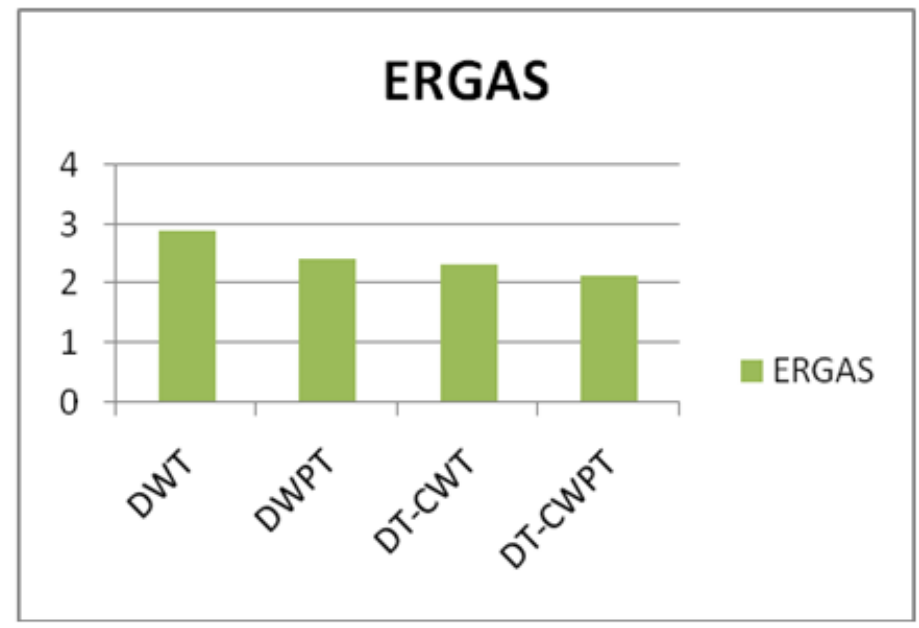

Fig. 7c ERGAS Value for Fusion Methods

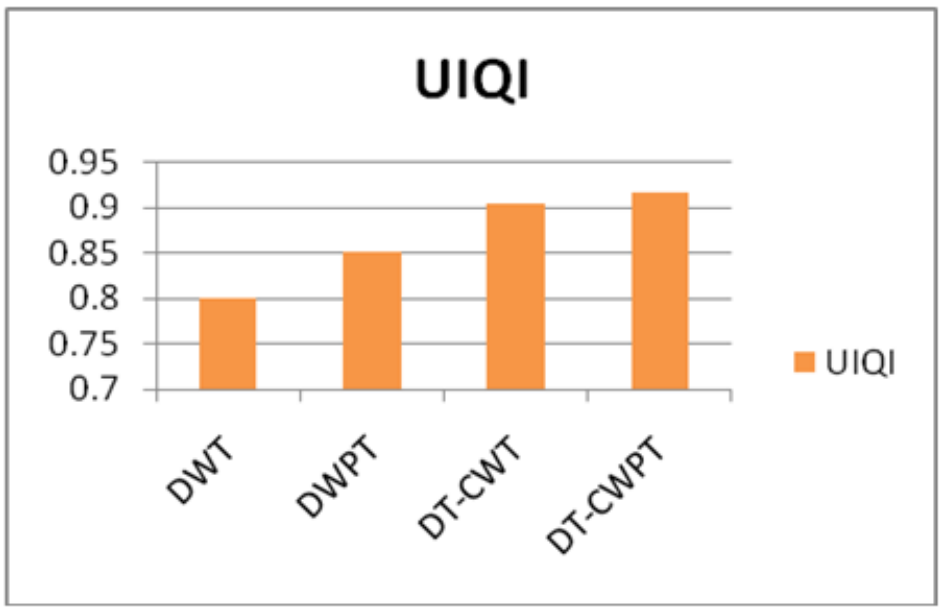

Fig. 7d. UIQI for Fusion Methods

From the visual inspection results and the quantitative results, it can be seen that the experimental results are in conformity with the theoretical analysis. When comparing the results, the CC of the proposed method is significantly higher which shows that it preserves the color of the MS image better than the other three fusion methods. The RMSE of the proposed fusion image shows a lower value than other methods which means the color distortion of the fused image is less. When the ERGAS value is compared, proposed method has the lowest value and is less than three which represents satisfactory quality. The UIQI for the proposed method is higher than other three methods and is nearest to one which shows a large amount of salient information is transferred to the fused image. Since, DT-CWPT allows the splitting of approximate as well as the detail coefficients; intuitively its performance is better than that of DT-CWT and other methods. So the proposed method produces the fused image closest to those the corresponding multi-sensors would observe at the high-resolution level.

\section{Conclusion}

This paper presents a novel approach using integration of DT-CWPT and HSV transform to fuse highresolution panchromatic image and low resolution multispectral image. To substantiate the results obtained, an evaluation is made using different performance metrics which compares the proposed method with the existing DWT, DWPT and DT-CWT methods. From the results and analysis, it is inferred that the proposed method shows better performance in terms of the high-quality synthesis of spectral information while retaining the spatial content of the pan image and it provides better result qualitatively and quantitatively than the other methods. This work infers that the fusion method using DT-CWPT and HSV offers computationally efficient image fusion technique and intends to focus more on complex wavelet packets based fusion techniques to improve the existing. 


\section{References}

[1]. Ivan W. Selesnick, Richard G. Baraniuk, and Nick G. Kingsbury "The Dual -Tree complex wavelet transform," IEEE signal processing magazine, Nov. 2005,pp.123-151

[2]. Edwards and P.A.Davis, "The use of Intensity-Hue-Saturation transformation for producing color shaded-relief images," Photogramm. Eng. Remote Sens., vol. 60, no. 11, 1994, pp. 1369-1374

[3]. P. D. Shukla, "Complex wavelet transforms and their applications", PhD Thesis, The University of Strathclyde, 2003.

[4]. N.G. Kingsbury, "Image processing with complex wavelets," Philos. Trans. R.Soc. London A, Math. Phys. Sci., vol. 357, no. 1760, Sept. 1999, pp. 2543-2560.

[5]. Peter de Rivaz and Nick Kingsbury, "Bayesian image deconvolution and denoising using complex wavelets", Proc. IEEE Conf. on Image Processing, Greece, Oct 2001, paper 2639

[6]. Fernandes, "Directional, shift-insensitive, complex wavelet transforms with controllable redundancy", PhD Thesis, Rice University, 2002.

[7]. Nick Kingsbury, "The dual_tree complex wavelet transform: A new efficient tool for image restoration and enhancement" Proc. European Signal Processing Conference, EUSIPCO 98, Rhodes, 1998, pp 319-322.

[8]. Wenbo W, Y.Jing, and K. Tingjun, "Study Of Remote Sensing Image Fusion And Its Application In Image Classification" The Int. Archives of the Photogrammetry, Remote Sensing and Spatial Information Sciences, Beijing ,Vol. XXXVI, Part B7, 2008, pp. 11411146

[9]. S G Mallat, “A theory for multiresolution signal decomposition: The wavelet representation”, IEEE Trans. PAMI, 11(7), 1989, pp 674-693

[10]. Chibani, Y., and A. Houacine, "The joint use of the HIS Trasform and the redundant wavelet decomposition for fusing multispectral and panchromatic images", International Journal of Remote Sensing, 23(18), 2002, pp 3821-3833.

[11]. J.G.Liu, "Smoothing filter-based intensity modulation:A spectral preserve image fusion technique for improving spatial details," Int. J. Remote Sens., vol. 21, no. 18, 2000, pp. 3461-3472.

[12]. T.M.Tu, S.C.Su, H.C.Shyu, and P.S.Huang, "A new look at IHS-like image fusion methods," Inf. Fusion, vol.2, no. 3, 2001, pp $177-186$.

[13]. J. N'u nez, X. Otazu, O. Fors, A. Prades, V. c Pal’a, and R.Arbiol, “Multiresolution-Based Image Fusion with Additive Wavelet Decomposition," IEEE Transactions on Geoscience and Remote Sensing, vol.37, 1999, pp.1204 - 1211.

[14]. T. Ranchin and L. Wald, "Fusion of high spatial and spectral resolution images: The ARSIS concept and its implementation," Photogramm. Eng. Remote Sens., vol. 66, no. 1, 2000, pp. 49-61.

[15]. B.Aiazzi, L.Alparone, S.Baronti, and A.Garzelli, "Context-driven fusion of high spatial and spectral resolution images based on over sampled multi-resolution analysis," IEEE Trans. Geosci. Remote Sens., vol. 40, no. 10, Oct. 2002, pp. $2300-2312$.

[16]. Y. Zhang, "A new merging method and its spectral and spatial effects," Int. J. Remote Sens., vol. 20, no. 10, 1999 , pp. $2003-2014$.

[17]. Ehlers M., S. Klonusa, P. Johan and P. Rosso, "Multi-sensor image fusion for pansharpening in remote sensing", International Journal of Image and Data Fusion, Vol. 1, No. 1, March 2010, pp. 25-45.

[18]. R. A. Schowengerdt, Remote Sensing: Models and Methods for Image Processing, 2nd ed. Orlando, FL: Academic, 1997.

[19]. Wang Z. and A.C. Bovik, "A universal image quality index," IEEE Signal Process Lett., 9(3), 2002, pp. 81-84.

[20]. K.Shivsubramani, P soman, Krishnamoorthy, "Implementation and Comparative Study of Image Fusion Algorithms", International Journal of Computer Applications (0975 - 8887) Volume 9, No.2, November 2010, pp.3-6.

[21]. Anjali Malviya and S.G.Bhirud ,"Image Fusion of Digital Images", Int. J. Recent Trends in Engineering, Vol.2, No.3, November 2009, pp. 2-4.

[22]. V.P.S Naidu and J.R.Raol, "Pixel level Image fusion using wavelets and Principal component analysis", Defence science Journal, Vol.58, No.3, May 2008, pp. 338-352.

[23]. A. Goshtasby and S. G. Nikolov, "Image fusion: Advances in the state of the art", Editorial-Science Direct, Special Issue on Image fusion, 8(2), April 2007, pp 114-118.

[24]. H.Wang, J. Peng, and W.Wu," Fusion algorithm for multisensor image based on discrete multi wavelet transform," IEEE Proc. Visual Image Signal Process., 149(5), 2002.

[25]. K. Amolins, Y. Zhang, and P. Dare, "Wavelet based image fusion techniques - An introduction, review and comparison," ISPRS Journal of Photogrammetry \& Remote Sensing, vol.62, 2007, pp. 249-263. 